\title{
Yoga verringert oxidativen Stress
}

Ein dreimonatiges Yoga-Programm zusätzlich zur Regelversorgung hat bei Typ-2-Diabetes viele positive Effekte. Das ergab einer kontrollierte klinische Studie mit 123 Diabetikern. Im Vergleich zur Kontrollgruppe sanken die Werte für oxidativen Stress. Zudem sank der BMI, der Taillenumfang aber nicht.

Die Patienten wurden auf die Gruppen stratifiziert verteilt Hegde SV, Adhikari P, Kotian S et al. Diabetes Care 2011;34 (10):2208-10. im Hinblick auf mikro- und makrovaskuläre Komplikationen, periphere Neuropathie, keine Komplikationen.
Ergebnisse: Im Vergleich zu den Patienten mit der ausschließlichen Regelversorgung führte das Yoga-Training bei den Teilnehmern zu einer signifikanten Verringerung von BMI, glykämischer Kontrolle und Malondialdehyd sowie zu einem Anstieg der Glutathion- und Vitamin C-Werte. Jedoch konnte im Studienverlauf in der Yoga-Gruppe kein Unterschied hinsichtlich des Taillenumfangs, des Taillen-Hüft-Verhältnisses, des Blutdrucks, der Vitamin E-Werte sowie der Superoxid-Dismutase festgestellt werden.

Quelle (3 Texte): www.lifestyle-telegramm.de

\section{Herzhaftes bremst Appetit mehr als Süßes}

Herzhafte Vorspeisen verringern den anschließenden Verzehr von herzhaften Speisen mit hohem Fettgehalt im Vergleich zu Vorspeisen mit süßem oder neutralem Geschmack. Das hat eine englische Studie mit 30 normalgewichtigen Frauen ergeben, die nach der Vorspeise an einem Test-Buffet Nahrungsmittel frei wählen und konsumieren durften. Wer zu übermäßiger Nahrungsaufnahme neigt, sollte also besser herzhaft starten.

Der Geschmack spielt bei der

Finlayson G, Bordes I, Griffioen-Roose $S$ et al. Pic J Nutr 2012;142(1):125-30.
Nahrungsaufnahme. Die Frauen erhielten eine Standard"Vorspeise“, die herzhaft, süß oder neutral schmeckte. Mit Angaben zum Hunger wurde die Sättigung durch die Vorspeise ermittelt, in einem Computer-Modell das Belohnungsgefühl und mit einem Fragebogen das sonstige Essverhalten.

Ergebnisse: Sättigung und Gesamtnahrungsaufnahme wurden durch den Geschmack der Vorspeise nicht beeinflusst, jedoch wirkte sich dieser auf das Mögen sowie die Auswahl der Speisen aus. Im Vergleich zu der süßen bzw. neutralen Vorspeise verringerte die herzhafte Vorspeise das Mögen bzw. den Verzehr von herzhaften Speisen mit hohem Fettgehalt.

\section{Senkung durch regelmäßigen Fischkonsum}

Regelmäßiger Fischkonsum senkt die Risiken für Herz-Kreislauf-Erkrankungen. Im Gegensatz zum Verzehr von Obst, Gemüse, alkoholischen Getränken und Milchprodukten reduzierte er die endotheliale Dysfunktion und subklinische Inflammation. Das ergab eine Studie über 6 Jahre mit 301 Teilnehmern. Schon früher konnte eine gesunde Ernährung mit einem hohen Gehalt an Fisch, Obst und Gemüse, einem moderaten Alkoholkonsum und geringem Verzehr von Milchprodukten mit ver-

Van Bussel BC, Henry RM, Schalkwijk CG et al. J Nutr 2011;141(9):1719-25. minderten Konzentrationen zirkulierender Biomarker der endothelialen Dysfunktion und der subklinischen Inflammation in Zusammenhang ge- bracht werden. Unbekannt ist, wie sich der Verzehr dieser Lebensmittel längerfristig darauf auswirkt. Mithilfe der „DietHistory“-Querschnittsmethode wurde die Ernährungsweise festgehalten. Jeweils im Alter von 36 und 42 Jahren wurden einer Reihe von Parametern der Gerinnung und Inflammation gemessen. Anhand dessen wurden Assoziationen gesucht.

Ergebnisse: Nach multipler Adjustierung konnte der Konsum von Fisch (pro $100 \mathrm{~g}$ /Woche) im Gegensatz zu allen anderen Lebensmittelgruppen invers mit Veränderungen der endothelialen Dysfunktion [ $\beta(95 \%-\mathrm{KI})=-0,06(-0,10 ;-0,02) ; \mathrm{p}=0,003]$ sowie der subklinischen Inflammation $[-0,05(-0,09 ;-0,003) ; \mathrm{p}$ $=0,036]$ assoziiert werden. 